\title{
建设项目环评报告编制的基本错误探讨
}

蒋世友

安徽合肥肥东县环境保护局

DOI:10.32629/as.v2i1.1523

[摘 要] 本文以建设项目环境影响评价作为对象, 简单介绍了建设项目环境影响评价过程、环评报告审批流程和环评报告的 基本组成,并结合环评报告的基本组成，针对环评报告编制的基本错误加以探讨，供环评报告编制技术人员引以为鉴，以提高环 评报告质量。

[关键词] 环境影响评价; 环评报告; 评价过程; 审批流程; 基本组成; 基本错误

根据环境影响评价法 ${ }^{[1]}$ 和建设项目环境影响评价分类 管理名录 ${ }^{[2]}$ 的要求, 目前大部分的建设项目均需编制建设项 目环境影响评价报告 (以下简称: 环评报告) 上报到相应的环 境保护主管部门进行审批后, 方可开工建设。建设项目环评 报告编制需遵循建设项目环境影响评价相关的法律、法规、 导则和技术方法等的要求, 故对环评报告编制技术人员的综 合业务能力要求较高。目前, 因为从事环境影响评价编制工 作的技术人员数量众多, 且大多数为刚参加工作缺乏经验的 年轻人, 人员业务能力差异较大, 故上报的环评报告存在大 量的基本错误。所谓基本错误是指环评报告中经常性、普遍 性存在的常规的、低级的错误, 与建设项目本身无关, 仅与环 评报告编制有关的错误。

\section{1 建设项目环境影响评价过程}

建设项目的环境影响评价过程主要包括: 环评委托、环 评报告编制、环评报告受理和环评报告审核审批等四个部 分: 首先由建设项目建设单位委托环评报告编制单位进行项 目环境影响评价, 环评报告编制单位接受委托后按要求进行 环评报告的编制, 环评报告编制完成后, 打印装订成册报送 到相应的环境保护主管部门进行受理, 再由环境保护主管部 门进行审核审批, 最终由环境保护主管部门下发项目批文, 建设单位获取批文后开工建设。

\section{2 建设项目环评报告审批流程}

建设项目的环评报告审批流程主要包括受理、审核和审 批三步: 环评报告报送后, 由环境保护主管部门受理, 并对环 评报告进行审核, 审核通过之后出项目批文, 并打印盖章后 下发给建设单位。

另外, 如果环境保护主管部门审核过程中, 发现建设项 目或环评报告存在重大问题时, 从环境影响的角度分析, 建 设项目的建设不可行, 则直接将环评报告退回, 不作受理, 也 不再作审批。

\section{3 建设项目环评报告基本组成及错误}

3. 1 环评报告基本组成

建设项目环评报告按项目类别的不同分为两类: 环境影 响评价报告书和环境影响评价报告表, 报告书与报告表的基 本组成相同, 主要包括: 报告正文、附件、附图和附表等四
个基本组成部分。

3.2 环评报告基本错误

3. 2.1 环评报告正本错误

3. 2. 1. 1 版面格式错误: 未按总纲 ${ }^{[3]}$ 的要求设置报告章 节层次; 排版的格式错误。

3.2.1.2 编制依据错误: 与项目不符, 未及时更新, 缺失 地方依据等。

3.2.1.3 “项目主要建设内容及规模一览表” 错误: 表 中的 5 大基本工程不全; 各项目归类划分错误; 建设内容不 全面; 与总平面布置图严重不符。

3.2.1.4 产品方案错误: 产品方案不清, 无规格描述, 且 与项目的生产原辅材料、生产设备、总平面布置图、产能、 生产工艺等不符。

3.2.1.5 主要原辅材料错误: 原辅材料表中缺原辅材功 用项, 且与项目的产品方案、生产设备、总平面布置图、产 能、生产工艺不符, 缺有毒有害原辅材料的理化性质分析与 一次最大存储量等内容及成分清单列表。

3. 2.1.6 主要生产设备错误: 主要生产设备表中缺设备 功能和安装位置项, 且与原辅材料、产品方案、总平面布置 图、产品产能、生产工艺等不符。

3.2.1.7 建设项目所在地自然环境简况调查错误: 报告 中含有社会简况介绍, 内容张冠李戴, 与项目无关。

3.2.1.8 环境质量现状评价错误: 引用了别的项目报告 的监测数据, 未进行引用数据的可行性、有效性分析; 缺监 测布点图; 监测布点不规范; 现状评价方法错; 项目的特征 因子未作现状监测或引用; 执行的质量标准错误。

3.2.1.9 环境保护目标错误: 环境保护目标不全, 有遗 漏; 未按相关导则要求列出保护目标。

3.2.1.10 评价适用标准错误: 环境质量与排放标准存 在遗漏或多余因子, 与现状评价、影响预测评价等对不上, 特别是项目的特征因子。

3.2.1.11 关于工程分析错误

任何一个项目, 工程分析是一切的中心, 运营期工艺流 程与产污节点图 (以下简称：流程图) 是报告的重点, 也是报 告中所有内容的关联节点, 如果工程分析有错误, 一切错误 
都可能出现, 也无法避免。

一般情况下环评报告中工程分析错误主要有:

(1) 缺少施工期工程分析: 如项目为新建项目, 包含有施 工期, 可工程分析中却无施工期工艺流程分析。

(2) 项目流程图与产品方案等不符: 如项目有两种或以 上产品方案, 可却只有一个流程图。

(3) 项目流程图与原辅材料不符: 如流程图中未包含所 有的原辅材料, 仅列出了最主要的一种原料或两种原料, 其 它的原辅材料均没有列入 (出现 “无用的原辅料”), 反之出 现原辅材料清单表中从未出现的原辅料 (出现的 “无中生有 的原辅料”)。

(4) 项目流程图与生产设备不符: 如流程图中出现了无 对应该生产设备的工艺工序, 反之出现了 “无用” 的设备; 再者, 设备的功用功能与工艺工序对应不上。

(5) 项目流程图与总平面布置图不符: 如流程图与项目 的总平面布置图中的相应的生产工艺工段、生产车间、生产 厂房对应不上, 两者互不相关, 各自独立。

（6）项目流程图与项目的污染物产生节点、类型不符: 如 流程图与相应的污染物无法对应; 再者污染物类别标识错误。

（7）项目流程图与生产工艺说明不符: 主要表现在生产 工艺说明不详细, 流程图与生产工艺说明不对应, 前后不一 致, 各自独立。

（8）项目流程图不规范: 如流程图中标识的工艺工序步骤 名称写成了设备名、原辅材料名; 整个流程图中无产污节点, 也无污染因子; 污染物产生的箭头应为虚线箭头, 却画成了实 线; 流程图采用截图, 把别的报告, 甚至是纸上的图截下来放在 报告中, 一则不清楚, 二则根本就不是本项目的, 未细化核实。

(9) 项目流程图与污染源、源强分析对应不上：如流程 图标识的污染源却无对应的污染源、源强分析内容, 同样所 分析的污染源、源强却无对应的工艺流程与产污节点, 典型 的前后不一致; 再者是项目的污源源强确定随意, 未进行细 化核实, 跟原辅材料用量、产能严重不符。

(10) 改扩项目流程图未按改扩建项目性质提供: 如应该 按改扩建项目的性质, 分别提供改建之前的现有项目的流程 图, 再提供改建之后的改扩建项目的流程图, 同样污染源分 析也应该按改扩建前后分别进行分析。

3.2.1.12 关于环境影响分析错误: 施工期环境影响分 析太泛泛, 没有针对性; 项目运营期环境影响分析缺项、漏 项严重; 项目环境影响分析中提出的环保措施太随意, 未作 深入的经济与技术可行性论证; 缺特殊影响的分析内容。

3.2.1.13 关于报告中其它错误

报告中的其它错误一般均较多, 如报告中内容前后不一 致, 甚至于同一个页面的上、下文之间的内容都不一致, 自相 矛盾; 报告的结论应该为 “从环境影响角度分析, 本项目的 建设是可行的”, 可多数报告写成了 “从环境保护角度而言”; 报告正文中的插图、插表的图题、表题错误较多; 文字上的 张冠李戴错误较多。报告中的版式错误, 如标题文字未加粗、
段落未对齐, 表格对齐错误, 单位与符号错误等等。再者, 报 告中的标题序号错误, 章节层次混乱。

3.2.1. 14 报告用词用语不当, 甚至出现名词概念性错 误: 如报告中将大气污染物“浓度贡献值”写成“增加浓度”, “单因子指数” 写成 “污染指数”, 另外口头语用作书面语, 如 “无” 写作 “没有” , “非” 写作 “不是”。

3.2 .2 环评报告附件错误

3.2.2.1 附件不全: 缺企业营业执照、土地证、规划环 评批复、引用监测数据原件扫描件、相关环评的批复及验收 报告等等, 甚至有的报告附件全无。

3.2.2.2 附件不规范: 盖章的附件应为彩色, 却为黑白 件、环评委托函未盖章和缺日期等、监测数据为 Word 版, 非监测报告扫描件, 监测报告中未附现状监测时的监测人员 采样工作照等。

3.2.2.3 附件不清楚: 如引用项目的监测报告、租房合同 不清楚, 无法看清楚其中内容, 则无法对内容的真实性作判断。

3.2. 3 环评报告附图错误

3.2.3.1 附图不全: 如缺园区规划图、项目周边关系图、 项目四邻现状图、敏感目标分布图、项目现状监测布点图、 项目分区防渗图等等; 引用监测数据的项目缺本项目与引用 项目的位置关系图; 改扩建项目缺改扩建之前的现有项目总 平面图和改扩建之后的总平面图, 多为两图混一图。

3.2.3.2 附图不规范: 附图中缺比例尺、风向玫瑰图、 图例; 总平面布置图中缺环保措施; 附图与报告内容不一致, 如总平面布置图中的内容跟项目的主要建设内容一览表对 应不上, 各个附图的风向玫瑰图不一致。

3.2.3.3 附图不清楚: 附图打印时, 打印灰度的图片, 不 清楚; 另外, 如项目的地理位置图采用地形图截图, 无法获知 项目具体位置。

3.2. 4 环评报告附表错误

3.2.4.1 格式错误: 未按生态环境部要求, 提供标准格式 的 Excel 表, 却提供了不符合要求的 Excel 表格, 甚至为 Word 表、PDF 表格等。

3.2. 4.2 填写内容不规范: 建设内容、规模填写不全; 表中的 “环境影响评价行业类别” 错, 未按最新的 “建设项目 环境影响评价分类管理名录 (2018版 4 月 28 日修订)”的类别 进行填写, 甚至填成了国民经济行业分类中的类别, 或者仅有 大类无小类, 或者仅有小类无大类; “国民经济行业类别” 填 写错, 未按 “2017 年国民经济行业分类 (GBT4754-2017)” 中的 类别代码填写; “污染物排放量” 中的内容填写不全; 建设单 位的信息填写错误；开工与投产时间填写错误等。

3.2.4.3 表格内容缺失: 缺园区规划环评的内容, 次之 是建设单位的 “统一社会信用代码”, 还有缺项目代码等等。

3.2.4.4 项目地理位置经纬度错误: 未以谷歌地球中的 项目地理位置坐标为准, 而是用了其它一一奥维、91 等, 根本 无法进行项目定位。

\section{4 结束语}

Copyright @ This word is licensed under a Commons Attibution-Non Commercial 4.0 International License. 


\section{水产养殖中禁用渔药的危害}

周风云

江苏省宿迁市沭阳县韩山镇人民政府

DOI:10.32629/as.v2i1.1513

[摘 要] 本文主要对水产养殖中禁用渔药的种类和危害进行了分析和阐述, 以期为水产养殖产量的增加, 保障水产品的安全 提供帮助。

[关键词] 水产养殖; 禁用鱼药; 危害; 安全

现阶段, 在我国渔药生产、销售以及使用过程中存在着较多 问题, 且相关管理体系的构建也不完善, 使得很多违禁药品被应 用在水产养殖中, 这不仅难以保证公众食用到放心的鱼产品, 也 阻碍了我国水产品的出口。基于此, 应加大水产养殖户对禁用渔 药的分辨力度, 明确禁用渔药的危害, 提高水产养殖效率。

\section{1 抗生素}

抗生素类药物是一种微生物合成制剂, 以白色针状晶体 形态出现, 微溶于水, 且水溶液呈中性。最常看到的抗生类药 物有氯霉素和红霉素两种。其中氯霉素在渔业生产中主要被 用来治疗赤鯺、红点病、弧菌病和痘疮病等; 而红霉素大多 被用来治疗白皮病和白头白嘴病等。不过应用该类药品的鱼 产品如果被人们食用, 会产生较大毒性, 很容易影响人体造 血功能的正常运转, 进而导致人们出现再生障碍性贫血、肠 道菌群失调等症状, 降低人体机能以及免疫力水平。

对此, 我国在抗生素类药品应用上已经制定了较为严格 的要求, 氯霉素制剂禁止被应用在食品或者动物中, 对于红 䨱素制剂, 其用量有着严格控制, 一般在水产品中的用量不 得超过每公斤 100 微克。另外, 为了降低抗生素类药品对水 产品和人类的危害, 还可以利用土霉素、四环素、金霉素、 噁咺酸等同等性能和功效的药品替代, 以保证水产品质量。

\section{2 有机氯制剂}

有机氯制剂一般呈现白色或者淡黄色块状晶体结构, 最常见 到的药剂种类有六氯化苯、林丹、毒杀芬、DDT 这四种, 是杀灭
鱼虫、水蜈蚣最为有效的武器。不过有机氯试剂自身具有的毒性 相对较高, 降解时间长, 在水生物中残留的时间较长, 很容易产生 生物富集现象。如果不小心误食这类产品, 会导致人体出现器官 衰竭, 增加患癌几率。所以该类药物早在 60 年代初期时就已经被 禁停销售和使用了。现阶段为了降低鱼禹、水蜈蚣等天敌的侵袭, 通常是利用有机磷制剂代替原有的有机氯制剂, 这不仅能够起到 消灭和预防的作用, 而且也不会对人体造成较大威胁。

\section{3 含录制剂}

含录制剂主要以甘录为主, 其构成成分有二氧化录、硝 酸亚录、醋酸录和吡定基醋酸录。该类药品呈现白色晶状颗 粒, 在加热后会生成贡元素。众所周知, 贡属于重金属的一种, 对于人、动物有着较大毒性。在水产养殖中, 含录制剂主要 被应用在解决小瓜虫病、车轮虫、斜管虫、白头白嘴病以及 白皮病等症状上, 不过由于其分解所产生的贡元素会存在较 大毒性, 如果被人体使用, 轻则会产生中毒反应, 重则会导致 新生儿童畸形、癌症以及突变, 严重威胁人类的生命安全。 据相关调查研究表明, 录元素积聚在人体后, 很难通过新陈 代谢排出体外。同样的其在鱼体中寄存, 也会因为生物链关 系逐渐转移到人体内, 进而造成录元素堆积, 影响各项器官 的运行。因此该类药机已经被我国明文禁止。现今水产养殖 中, 会利用福尔马林来代替原有的含录制剂进行小瓜虫病的 治疗, 另外还有养殖户会采用干辣椒粉、生姜、五倍子和土荆 芥配置而成的中草药来替代含录药剂, 达到虫害的治疗效果。
目前, 环境保护主管部门需受理大量的建设项目环评报告, 绝大多数的建设项目本身是符合国家的环保政策的, 从环境影 响角度分析, 项目的建设是可行的。但由于所报上来的建设项目 环评报告存在大量的基本错误, 造成环评报告的质量低下, 评价 结论不可信, 环境保护主管部门无法进行审批, 企业无法获批开 工建设, 给国家、地方的经济建设和社会发展造成了巨大的负面 影响。因此, 针对环评报告的基本错误, 作为建设项目环评报告 编制技术人员应与时俱进, 勤于学习, 时刻掌握最新的环评报告 编制要求, 牢牢掌握基本的环评报告编制技巧, 认真细心地进行 环评报告的编制, 尽量避免出现基本错误, 力求尽可能地提高环 评报告质量, 减轻环境保护主管部门的审批压力, 为建设单位争 取尽早获取项目批文, 力争建设项目尽快开工建设而努力, 为国
家、地方的经济建设和社会发展作出应有的贡献。

\section{参考文献:}

[1]贾宝亮.浅析建设项目环境影响评价分类管理制度 [J].天津科技,2010,37(04):29-31.

[2]刘殊,姜华,梁鹏.优化评价内容提高环评效能- 《环境影响评价技术导则总纲》修订思考与建议[J].环境影 响评价,2016,38(06):28-30.

[3]陆建虹, 付君伟。通过碳足迹和水足迹评价铝车身对 环境影响 [J].汽车工业研究,2019,(01):45-48.

作者简介:

蒋世友(1975--) 男, 汉族, 安徽合肥人, 本科学历, 工程师, 主要从事环境保护工作。 